\title{
Health care on mobile devices
}

\author{
Guy Pujolle ${ }^{1}$
}

Published online: 12 August 2016

(C) Institut Mines-Télécom and Springer-Verlag France 2016

Health is perhaps the area that will be the most important in the networking field in a decade. All large industrial groups are preparing products to check and treat us in continuous time: Apple, Google, Samsung, etc. have already issued medical applications. Different types of networking research paradigms will be represented from wireless sensors networks or body area networks (BAN) to Cloud networking. The sensors inside the body or on its surface will generate data flows that will be interpreted either locally or remotely. Fog and Cloud networking and computing will play an important role through diverse analysis methods such as big data analytics. The goal is clearly to monitor billions of people continuously with decision-making. The idea is to warn the patient of possible problems detected by the sensors and corroborated by other factors determined from Cloud analysis.

Works to perform in this area are numerous and cover a quite large field. In particular, security schemes are definitely necessary to networks and Cloud environments which must manage and control valuable data. The global system must take care of the privacy and should highlight all the problems that may arise.

This special issue on health care on mobile devices aims precisely to get around all these problems. A severe selection was made to select very good position papers. Major issues are addressed as co-existence of BAN and LAN, security, quality of service, and Cloud management.

More specifically, seven papers have been accepted. Two are dealing with interferences between BANs and between BANs and LANs. The first one on "Performance evaluation

Guy Pujolle

guy.pujolle@lip6.fr

Lip6, UPMC/CNRS, Paris, France of co-located IEEE 802.15.4-based wireless body sensor networks" by Amirhossein Moravejosharieh and Jaime Lloret is considering a situation in which a large number of people wearing body sensor networks are gathered in very close vicinity. Two types of interference mitigation strategies are proposed: passive and active schemes.

The second one entitled "Fairness-aware radio resource management for medical interoperability between WBAN and WLAN" by Byeong-Moon Cho, Kyung-Joon Park, and Eun-Chan Park proposes a novel channel access protocol for achieving effective channel sharing with respect to efficiency and fairness. This proposal makes it possible to control the contention window size of WLAN based on the delay information of WBANs.

Another area is examined through the third paper of this special issue entitled "Performance of IR-UWB cross-layer ranging protocols under on-body channel models with body area networks" co-authored by Arturo Guizar, Claire Goursaud, and Jean-Marie Gorce. This paper deals with the performance of sensors positioning through the 3-Way ranging protocol (3-WR) in a wireless body area network (WBAN). The purpose is to propose a new cooperative algorithm to increase the localization of the WBAN nodes.

The field of security is also well represented in this special issue through two papers. The first one, "A differential privacy protection scheme for sensitive big data in body sensor networks" by Chi Lin, Pengyu Wang, Houbing Song, Yanhong Zhou, and Qing Liu.Guowei Wu proposes a differential privacy protection scheme for sensitive big data in body sensor networks. A tree structure is constructed to reduce errors and provide long-range queries. The second paper deals with a "Performance Evaluation of Enhanced Very Fast Decision Tree (EVFDT) mechanism for distributed denial-of-service attack detection in health care systems" by Haider Abbas, Rabia Latif, Seemab Latif, and Ashraf Masood. A real-time 
Cloud-assisted WBAN testbed is deployed to investigate the efficiency and accuracy of proposed algorithms for real-time sensor network traffic.

The last two papers of this special issue concern network mobility and Cloud processing. The first one entitled "A network-assisted flow mobility architecture for optimized mobile medical multimedia transmission" by Norbert Varga, Lasz Bokot, and Esa Piri deals with advanced and reliable mobile communication solutions to provide efficient multimedia transmission with strict medical level Quality of Service (QoS) and Quality of Experience (QoE) provision. The paper proposes a network-assisted flow-based mobility management architecture for optimized real-time mobile medical multimedia communication.

Finally, the last paper entitled "Cloud support for large scale e-healthcare systems" by Qutaibah Althebyan, Qussai Yaseen, Yaser Jararweh, and Mahmoud Al-Ayyoub puts for- ward a large-scale e-healthcare monitoring system that targets a crowd of individuals in a wide geographical area. The system is efficiently integrating many emerging technologies such as mobile computing, edge computing, wearable sensors, Cloud computing, big data techniques, and decision support systems.

Finally, I would like to thank the three guest editors who have done an excellent job at selecting and offering us a high-quality issue in an area that will become increasingly important. All the ingredients to get a great success are gathered in this special issue. I wish a good reading to all who will have this special issue of Annals of Telecommunications in hands.

Guy Pujolle

Editor-in-Chief of Annals of Telecommunications

Lip6, UPMC/CNRS, Paris, France 